\title{
If Many Seek, Ye Shall Find: Search Externalities and New Goods
}

\author{
Maciej H. Kotowski* Richard. J. Zeckhauser ${ }^{\dagger}$
}

Online Appendix

\section{A Omitted Proofs}

\section{A.1 Preliminary Lemmas}

Lemma A.1. Consider a type- $v_{i}$ consumer who has access to contract $\sigma_{j}$. Suppose this consumer believes that all firms not yet sampled are following the same independent production/pricing strategy, $\tilde{\psi} \in \Delta(\Sigma)$. Define $u^{*}\left(v_{i}\right)$ as the unique solution $t^{1}$

$$
u^{*}\left(v_{i}\right)=\mathbb{E}_{\tilde{\psi}}\left[\max \left\{u^{*}\left(v_{i}\right), u\left(\sigma \mid v_{i}\right), 0\right\}\right]-s .
$$

1. If $\max \left\{u\left(\sigma_{j} \mid v_{i}\right), 0\right\} \geq u^{*}\left(v_{i}\right)$, then it is optimal for consumer $i$ to accept $\sigma_{j}$ (or, to exit the market if $\left.0>u\left(\sigma_{j} \mid v_{i}\right)\right)$.

2. If $u^{*}\left(v_{i}\right)>\max \left\{u\left(\sigma_{j} \mid v_{i}\right), 0\right\}$, then it is optimal for consumer $i$ to learn the contract offer of the next firm at a cost of $s$.

Proof. Consumer $i$ 's decision problem is essentially one of sequentially sampling from a stationary distribution of independently and identically distributed random variables. The value of each sampled contract is the random variable $\left[u\left(\sigma \mid v_{i}\right) \vee 0\right]$. (Each sample's value is bounded below by zero since a consumer may opt out of the market.) The distribution of this value is derived from the distribution of $\sigma$, which the consumer believes to be $\tilde{\psi} \in \Delta(\Sigma)$. Since prices are non-negative, the expectation of $\left[u\left(\sigma \mid v_{i}\right) \vee 0\right]$ exists. By DeGroot (1970, Sections 13.5 and 13.9), the optimal strategy for the consumer is the above cutoff rule.

${ }^{*}$ John F. Kennedy School of Government, Harvard University, 79 JFK Street, Cambridge MA 02138. E-mail: maciej_kotowski@hks.harvard.edu.

${ }^{\dagger}$ John F. Kennedy School of Government, Harvard University, 79 JFK Street. E-mail: richard_zeckhauser@harvard.edu.

${ }^{1}$ The notation in (A.1) is self-explanatory. Here, " $\sigma$ " is random. Its distribution is $\tilde{\psi}$. 
Lemma A.2. Suppose there exists an equilibrium where all firms adopt the strategy $\psi^{*}$. Let $u^{*}\left(v_{i}\right)$ be the cutoff value describing a type- $v_{i}$ consumer's equilibrium search strategy. Let $\sigma^{*}$ be a contract such that:

1. $u\left(\sigma^{*} \mid \bar{v}\right)>\max \left\{u^{*}(\bar{v}), 0\right\}$ and $u\left(\sigma^{*} \mid \underline{v}\right)>\max \left\{u^{*}(\underline{v}), 0\right\}$; or,

2. $u\left(\sigma^{*} \mid \bar{v}\right)>\max \left\{u^{*}(\bar{v}), 0\right\}$ and $\max \left\{u^{*}(\underline{v}), 0\right\}>u\left(\sigma^{*} \mid \underline{v}\right)$; or,

3. $\max \left\{u^{*}(\bar{v}), 0\right\}>u\left(\sigma^{*} \mid \bar{v}\right)$ and $u\left(\sigma^{*} \mid \underline{v}\right)>\max \left\{u^{*}(\underline{v}), 0\right\}$.

Then $\sigma^{*}$ is not in the support of $\psi^{*}$. That is, there exists an alternative contract that gives firm $j$ strictly greater profits than $\sigma^{*}$ given consumers' search behavior.

Proof. We give the proof for case 1 with the remaining cases following analogously. Suppose the conditions of the lemma are satisfied and that firm $j$ posts $\sigma^{*}$. This contract is accepted by both types of consumers whenever they encounter firm $j$. Suppose firm $j$ offers instead the contract $\sigma_{\epsilon}^{*}$, which is identical to $\sigma^{*}$ except that it sets a price that is $\epsilon>0$ greater. Suppose $\epsilon$ is sufficiently small so that $u\left(\sigma^{*} \mid \bar{v}\right)>u\left(\sigma_{\epsilon}^{*} \mid \bar{v}\right)>\max \left\{u^{*}(\bar{v}), 0\right\}$ and $u\left(\sigma^{*} \mid \underline{v}\right)>$ $u\left(\sigma_{\epsilon}^{*} \mid \underline{v}\right)>\max \left\{u^{*}(\underline{v}), 0\right\}$. Clearly, $\sigma_{\epsilon}^{*}$ is accepted by both types of consumers whenever $\sigma^{*}$ was. Hence $\sigma_{\epsilon}^{*}$ is accepted by the same expected number of consumers as $\sigma^{*}$. Since this number is strictly greater than zero and since $\sigma_{\epsilon}^{*}$ is associated with a strictly higher price, it will generate greater expected profits than $\sigma^{*}$. Therefore, $\sigma^{*}$ cannot be a contract that is offered in equilibrium.

Lemma A.3. Let $u^{*}\left(v_{i}\right)$ be the cutoff value characterizing a consumer's search strategy. (1) $u^{*}(\bar{v}) \geq u^{*}(\underline{v})$. (2) If $u^{*}\left(v_{i}\right) \geq 0$, then there exists some $\sigma$ in the support of $\tilde{\psi}$ such that $u\left(\sigma \mid v_{i}\right)>u^{*}\left(v_{i}\right)$.

Proof. Suppose $\sigma \sim \tilde{\psi}$. To simplify notation, define the real-valued random variable $X_{v_{i}} \stackrel{d}{=}$ $\left[u\left(\sigma \mid v_{i}\right) \vee 0\right]$. Let $F_{v_{i}}(x)$ be the associated distribution function. Since $u(\sigma \mid \bar{v}) \geq u(\sigma \mid \underline{v})$ for all $\sigma, X_{\bar{v}}$ first-order stochastically dominates $X_{\underline{v}}$. Hence $F_{\underline{v}}(x) \geq F_{\bar{v}}(x)$ for all $x$. Finally, we can write $u^{*}\left(v_{i}\right)=\int \max \left\{u^{*}\left(v_{i}\right), x\right\} d F_{v_{i}}-s$. For this equality to hold, $F_{v_{i}}\left(u^{*}\left(v_{i}\right)\right)<1$.

1. We argue by contradiction. Suppose $u^{*}(\bar{v})<u^{*}(\underline{v})$. Since $X_{\bar{v}}$ first-order stochastically dominates $X_{\underline{v}}, u^{*}(\underline{v})=\int \max \left\{u^{*}(\underline{v}), x\right\} d F_{\underline{v}}-s \leq \int \max \left\{u^{*}(\underline{v}), x\right\} d F_{\bar{v}}-s$. Thus,

$$
\begin{aligned}
u^{*}(\underline{v})-u^{*}(\bar{v}) & \leq \int \max \left\{u^{*}(\underline{v}), x\right\} d F_{\bar{v}}-\int \max \left\{u^{*}(\bar{v}), x\right\} d F_{\bar{v}} \\
& \leq \int\left(u^{*}(\underline{v})-u^{*}(\bar{v})\right) \mathbf{1}\left(x \leq u^{*}(\bar{v})\right) d F_{\bar{v}} \\
& \leq\left(u^{*}(\underline{v})-u^{*}(\bar{v})\right) F_{\bar{v}}\left(u^{*}(\underline{v})\right) \\
& \leq\left(u^{*}(\underline{v})-u^{*}(\bar{v})\right) F_{\underline{v}}\left(u^{*}(\underline{v})\right)
\end{aligned}
$$

Since $F_{\underline{v}}\left(u^{*}(\underline{v})\right)<1$, we arrive at a contradiction. Therefore, $u^{*}(\bar{v}) \geq u^{*}(\underline{v})$.

2. Again we proceed by contradiction. Suppose $u\left(\sigma \mid v_{i}\right) \leq u^{*}\left(v_{i}\right)$ for all $\sigma$ in the support of $\tilde{\psi}$. Then, $u^{*}\left(v_{i}\right)=\mathbb{E}_{\tilde{\psi}}\left[\max \left\{u^{*}\left(v_{i}\right), u\left(\sigma \mid v_{i}\right), 0\right\}\right]-s \leq \mathbb{E}_{\tilde{\psi}}\left[\max \left\{u^{*}\left(v_{i}\right), u^{*}\left(v_{i}\right), 0\right\}\right]-s=$ $u^{*}\left(v_{i}\right)-s$, which is a contradiction since $s>0$. 


\section{A.2 Proof of Theorem 1}

Lemma A.4 is used in the proof of Theorem 1, which follows.

Lemma A.4. There does not exist an equilibrium where only good 1 is produced.

Proof. We divide the argument into three cases. Throughout we assume that if a firm produces good 1 , it sets a price in the interval $[c, \bar{v}]$. Prices below $c$ and above $\bar{v}$ lead to negative profits.

1. Suppose there exists an equilibrium where all firms offer $\sigma^{*}=\langle 1, p\rangle, p \in[c, \bar{v}]$. Since all firms are offering the same contract, $u^{*}\left(v_{i}\right)=\max \left\{u\left(\sigma^{*} \mid v_{i}\right)-s,-s\right\}$ for $v_{i} \in\{\underline{v}, \bar{v}\}$. There are two sub-cases, each generating a contradiction.

(a) Suppose $p \leq \underline{v}$. If $p<\underline{v}$, then $u\left(\sigma^{*} \mid \underline{v}\right)>\max \left\{u^{*}(\underline{v}), 0\right\}$ and $u\left(\sigma^{*} \mid \bar{v}\right)>\max \left\{u^{*}(\bar{v}), 0\right\}$. By Lemma A.2 this is a contradiction. Hence, $p=\underline{v}$. Therefore, the profits of a typical firm must be $\underline{v}-c-\phi$.

Suppose firm $j$ offers instead the contract $\langle 0, z\rangle$. This contract is acceptable to all type- $\underline{v}$ consumers and firm $j$ ensures itself an expected profit of at least $(1-\gamma) z$. By Assumption A-1, $(1-\gamma) z>\underline{v}-c-\phi$. Hence, this alternative contract is more profitable - a contradiction.

(b) Suppose instead that $p \in(\underline{v}, \bar{v}]$. By reasoning analogous to the preceding case we conclude that $p=\bar{v}$. Thus, a typical firm's profit $\gamma(\bar{v}-c)-\phi$. Now, the contract $\langle 0, z\rangle$ is acceptable by both types of consumer and will yield an expected profit of $z$. Noting Assumption A-1, $z>\gamma(\bar{v}-c)-\phi$. Again, this is a contradiction.

2. Suppose there exists an equilibrium with price dispersion where all firms produce good 1 but consumers do not search. Thus, each firm sells its product to at most one consumer.

Suppose $\sigma=\langle 1, p\rangle$ and $\hat{\sigma}=\langle 1, \hat{p}\rangle, c \leq p<\hat{p} \leq \bar{v}$, are two contracts available in equilibrium. Since a type- $\bar{v}$ consumer does not search, $\max \left\{u^{*}(\bar{v}), 0\right\} \leq u(\hat{\sigma} \mid \bar{v})<$ $u(\sigma \mid \bar{v})$. If $\max \left\{u^{*}(\underline{v}), 0\right\} \leq u(\hat{\sigma} \mid \underline{v})$, then both contracts would be accepted by both types of consumers on the equilibrium path. Such an outcome is not compatible with profit equalization across contracts in equilibrium. Hence, $u(\hat{\sigma} \mid \underline{v})<\max \left\{u^{*}(\underline{v}), 0\right\} \leq$ $u(\sigma \mid \underline{v})$.

If $\max \left\{u^{*}(\underline{v}), 0\right\}<u(\sigma \mid \underline{v})$, then Lemma A.2 implies a contradiction. Thus, $\max \left\{u^{*}(\underline{v}), 0\right\}=$ $u(\sigma \mid \underline{v})$. Similarly, $\max \left\{u^{*}(\bar{v}), 0\right\}=u(\hat{\sigma} \mid \bar{v})$. Since the preceding reasoning applies to all pairs of contracts there are at most two contracts available in this equilibrium.

If $u^{*}(\underline{v}) \geq 0$, then by Lemma A.3 there must exist some available contract, say $\tilde{\sigma}=$ $\langle 1, \tilde{p}\rangle$, such that $u(\tilde{\sigma} \mid \underline{v})>u^{*}(\underline{v})$. But this is a contradiction. Hence, $u^{*}(\underline{v})<0$. Therefore, $u(\sigma \mid \underline{v})=0$, which implies $p=\underline{v}$. Thus, the expected profit of a typical firm in equilibrium is $\underline{v}-c-\phi<(1-\gamma) z$. But then any firm offering $\sigma$ can increase its expected profit by posting $\langle 0, z\rangle$ instead - a contradiction. 
3. Suppose there exists an equilibrium with price dispersion where all firms produce good 1 but (some) consumers do search on the equilibrium path. If a consumer ever searches, he will certainly also search whenever he matches with the most expensive contract in the economy. Let $\hat{\sigma}=\langle 1, \hat{p}\rangle$ be that contract. Note that both types of consumers cannot search on the equilibrium path after matching with $\hat{\sigma}$. Otherwise, any firm offering it would earn strictly negative profits. Hence, there are two cases.

(a) Suppose a type- $\underline{v}$ consumer searches on the equilibrium path. Thus, $u^{*}(\underline{v}) \geq 0$ and $u(\hat{\sigma} \mid \underline{v}) \leq u^{*}(\underline{v})$. Since $\hat{\sigma}$ must be acceptable to some type of consumer, $\max \left\{u^{*}(\bar{v}), 0\right\} \leq u(\hat{\sigma} \mid \bar{v})$. Since $u^{*}(\underline{v}) \geq 0$, by Lemma A.3 there exists some contract in the support of the firm's strategy, $\sigma=\langle 1, p\rangle$, such that $u^{*}(\underline{v})<u(\sigma \mid \underline{v})$. But, without loss of generality, this also implies $0 \leq u^{*}(\underline{v}) \leq u^{*}(\bar{v})<u(\sigma \mid \bar{v})$, which contradicts Lemma A.2.

(b) Suppose a type- $\bar{v}$ consumer searches on the equilibrium path. Thus, $u^{*}(\bar{v}) \geq 0$, $u(\hat{\sigma} \mid \bar{v}) \leq u^{*}(\bar{v})$, and $\max \left\{u^{*}(\underline{v}), 0\right\} \leq u(\hat{\sigma} \mid \underline{v})$. By Lemma A.3 there exists some contract in the support of the firm's strategy, $\sigma=\langle 1, p\rangle$, such that $u^{*}(\bar{v})<u(\sigma \mid \bar{v})$. But this also implies $\max \left\{u^{*}(\underline{v}), 0\right\}<u(\sigma \mid \underline{v})$. Again, we contradict Lemma A.2.

The three cases considered are exhaustive of the situations that may occur if only good 1 is produced.

Proof of Theorem 1. We first argue that the proposed situation is an equilibrium. If every firm offers $\sigma^{*}=\langle 0, z\rangle$ and consumers believe this to be the case, no consumer searches. Firm profits are $z$. No firm can gain by offering $\langle 0, p\rangle, p<z$. Since no consumer is searching, this alternative contract implies lower profits. If instead a firm offers $\langle 1, p\rangle$, its expected profits are bounded above by $\max \{\underline{v}-c-\phi, \gamma(\bar{v}-c)-\phi\}<z$ (Assumption A-1). Hence, this too is not a profitable deviation. Therefore, the market is in equilibrium.

Lemma A.4 shows that there cannot exist an equilibrium where only good 1 is produced. Thus, to establish uniqueness it is sufficient to show that there is no other equilibrium where only good 0 is produced. Let $\psi^{*}$ be the equilibrium strategy of a typical firm in a symmetric equilibrium where only good 0 is produced. In this equilibrium, each firm must earn strictly positive profits. To see this fact, note that firm $j$ can offer the contract $\sigma_{j}=\langle 0, \epsilon\rangle$ where $0<\epsilon<s$. Even if consumer $i=j$ believed all other firms were offering good 0 at a price of zero, he would still prefer to accept $\sigma_{j}$ without any search. This gives firm $j$ a profit of at least $\epsilon$. Since a firm's equilibrium profit is strictly greater than zero, every contract in the support of $\psi^{*}$ must satisfy $u\left(\sigma \mid v_{i}\right) \geq \max \left\{u^{*}\left(v_{i}\right), 0\right\}$. (Note that $u^{*}(\bar{v})=u^{*}(\underline{v})$ since only good 0 is available.) By Lemma A.2, this implies $u\left(\sigma \mid v_{i}\right)=\max \left\{u^{*}\left(v_{i}\right), 0\right\}$. Hence, there is at most one contract offered in this equilibrium. But this implies $u\left(\sigma \mid v_{i}\right)=0$ and so $z=p$.

\section{A.3 Proof of Theorem 2}

Theorem 2 is proved by Lemmas A.5-A.12. In each lemma we assume that there exists a symmetric equilibrium with product differentiation. $\psi^{*}$ denotes the equilibrium strategy of 
a typical firm. $\mathcal{C}_{k}$ is the set of contracts in the support of $\psi^{*}$ that provide good $k$.

Lemma A.5. In an equilibrium with product differentiation, $u^{*}(\bar{v}) \geq 0$.

Proof. Suppose $0>u^{*}(\bar{v})$. By Lemma A.3, $0>u^{*}(\bar{v}) \geq u^{*}(\underline{v})$. No consumer finds it worthwhile search. Any firm offering $\langle 0, p\rangle$ will have an incentive to increase $p$ until $p=z$ since both types of consumers accept $\langle 0, p\rangle$ for all $p \leq z$. Hence, in equilibrium each firm earns $z$ in profits. Since no consumers search, the maximal expected profits of a firm offering good 1 are bounded above by $\max \{\gamma(\bar{v}-c)-\phi, \underline{v}-c-\phi\}<z$, contradicting the equality of expected profits across equilibrium contracts.

Lemma A.6. There exists $\sigma^{*} \in \mathcal{C}_{1}$ such that $u\left(\sigma^{*} \mid \bar{v}\right)>u^{*}(\bar{v})$.

Proof. Suppose the contrary. If $u(\sigma \mid \bar{v}) \leq u^{*}(\bar{v})$ for all $\sigma \in \mathcal{C}_{1}$, then by Lemma A.3 there exists $\tilde{\sigma} \in \mathcal{C}_{0}$ such that $u(\tilde{\sigma} \mid \bar{v})>u^{*}(\bar{v}) \geq 0$. Since $u(\tilde{\sigma} \mid \bar{v})=u(\tilde{\sigma} \mid \underline{v})$ and $u^{*}(\bar{v}) \geq u^{*}(\underline{v})$, $u(\tilde{\sigma} \mid \underline{v})>\max \left\{u^{*}(\underline{v}), 0\right\}$. But this contradicts Lemma A.2.

Lemma A.7. There exists $\sigma^{*} \in \mathcal{C}_{1}$ such that $u\left(\sigma^{*} \mid \underline{v}\right) \geq \max \left\{u^{*}(\underline{v}), 0\right\}$.

Proof. Suppose $\max \left\{u^{*}(\underline{v}), 0\right\}>u(\sigma \mid \underline{v})$ for all $\sigma \in \mathcal{C}_{1}$. By Lemmas A.5 and A.6 there exists $\tilde{\sigma} \in \mathcal{C}_{1}$ such that $u(\tilde{\sigma} \mid \bar{v})>\max \left\{u^{*}(\bar{v}), 0\right\}$. But then $\max \left\{u^{*}(\underline{v}), 0\right\}>u(\tilde{\sigma} \mid \underline{v})$, which contradicts Lemma A.2.

Lemma A.8. The expected profit of a firm in an equilibrium with product differentiation is strictly positive.

Proof. Negative profits cannot be a feature of equilibrium. Thus, suppose they are zero. This implies that every contract of the form $\langle 0, p\rangle, 0<p<z$ must not be acceptable to either type of consumer. In particular, this implies that $0<z \leq u^{*}(\underline{v})$.

By Lemma A.3, there exists a contract, $\sigma^{*}$, offered in equilibrium such that $u^{*}(\underline{v})<$ $u\left(\sigma^{*} \mid \underline{v}\right)$. If $u^{*}(\bar{v})<u\left(\sigma^{*} \mid \bar{v}\right)$, then we would contradict Lemma A.2. Hence $u^{*}(\bar{v}) \geq u\left(\sigma^{*} \mid \bar{v}\right)$. There are now two possibilities.

1. If $u^{*}(\bar{v})=u\left(\sigma^{*} \mid \bar{v}\right)$, then by Lemma A.3 there exists an alternative contract available in equilibrium, $\sigma^{* *}$, such that $u\left(\sigma^{* *} \mid \bar{v}\right)>u^{*}(\bar{v})$. But in this case a type- $\underline{v}$ consumer would also benefit from $\sigma^{* *}$. Thus, $u\left(\sigma^{* *} \mid \underline{v}\right)>u^{*}(\underline{v})$, contradicting Lemma A.2.

2. If $u^{*}(\bar{v})>u\left(\sigma^{*} \mid \bar{v}\right)$, then we contradict Lemma A.2 immediately.

In either case we arrive at a contradiction.

Lemma A.9. For all $\sigma \in \mathcal{C}_{0}, u(\sigma \mid \underline{v}) \geq \max \left\{u^{*}(\underline{v}), 0\right\}$.

Proof. Suppose there exists $\sigma \in \mathcal{C}_{0}$ such that $\max \left\{u^{*}(\underline{v}), 0\right\}>u(\sigma \mid \underline{v})$. Since $u(\sigma \mid \bar{v})=$ $u(\sigma \mid \underline{v})<\max \left\{u^{*}(\underline{v}), 0\right\} \leq u^{*}(\bar{v}), \sigma$ is not accepted by any consumers. Therefore, the profits of a firm offering $\sigma$ must be zero contradicting Lemma A.8.

Lemma A.10. $\left|\mathcal{C}_{0}\right| \leq 2$. 
Proof. Let $\sigma=\langle 0, p\rangle$ and $\hat{\sigma}=\langle 0, \hat{p}\rangle$ and suppose $\sigma, \hat{\sigma} \in \mathcal{C}_{0}$. Without loss of generality, suppose $0<p<\hat{p} \leq z$. By Lemma A.9, $u(\sigma \mid \underline{v})>u(\hat{\sigma} \mid \underline{v}) \geq \max \left\{u^{*}(\underline{v}), 0\right\}$. Since both contracts must generate the same expected profits, $\hat{\sigma}$ must not be accepted by all type- $\bar{v}$ consumers. Thus, $u^{*}(\bar{v}) \geq u(\hat{\sigma} \mid \bar{v})$.

Suppose $u^{*}(\bar{v})=u(\hat{\sigma} \mid \bar{v})$. If in equilibrium a type- $\bar{v}$ consumer does not accept $\hat{\sigma}$ with probability 1 , then any firm offering $\hat{\sigma}$ can decrease its price by $\epsilon>0$, and induce all type- $\bar{v}$ consumers to accept. This infinitesimal decrease in price leads to a discrete increase in demand and would be a profitable deviation. Therefore, $u^{*}(\bar{v})>u(\hat{\sigma} \mid \bar{v})$. If $u(\hat{\sigma} \mid \underline{v})>$ $\max \left\{u^{*}(\underline{v}), 0\right\}$, then there would be a contradiction with Lemma A.2. Thus,

$$
u(\hat{\sigma} \mid \underline{v})=\max \left\{u^{*}(\underline{v}), 0\right\} .
$$

Now consider $\sigma$. If $u(\sigma \mid \bar{v})>u^{*}(\bar{v})$, then again Lemma A.2 is contradicted. If $u(\sigma \mid \bar{v})<$ $u^{*}(\bar{v})$, then it is only accepted by type- $\underline{v}$ consumers - contradicting the equality of profits between $\sigma$ and $\hat{\sigma}$. Therefore,

$$
u(\sigma \mid \bar{v})=u^{*}(\bar{v}) .
$$

Therefore, whenever there are at least two distinct contracts in $\mathcal{C}_{0}$, they must satisfy (A.2) and (A.3). Since these equations must hold for any pair of distinct contracts in $\mathcal{C}_{0}$, there are at most two elements in $\mathcal{C}_{0}$.

Lemma A.11. For all $\sigma \in \mathcal{C}_{1}, u(\sigma \mid \bar{v}) \geq u^{*}(\bar{v})$.

Proof. We argue by contradiction. Suppose there exists $\hat{\sigma} \in \mathcal{C}_{1}$ such that $u(\hat{\sigma} \mid \bar{v})<u^{*}(\bar{v})$. Without loss of generality we may assume that $\hat{\sigma}$ is the most expensive contract in $\mathcal{C}_{1}$. If type- $\bar{v}$ consumers do not accept $\hat{\sigma}$, type- $\underline{v}$ consumers must find it acceptable, $u(\hat{\sigma} \mid \underline{v}) \geq$ $\max \left\{u^{*}(\underline{v}), 0\right\}$; else, a firm would earn negative profits. If the preceding inequality is strict, Lemma A.2 would be violated. Hence, $u(\hat{\sigma} \mid \underline{v})=\max \left\{u^{*}(\underline{v}), 0\right\}$. Since $\hat{\sigma}$ was the most expensive contract in $\mathcal{C}_{1}$, a type- $\underline{v}$ consumer must accept all contracts in $\mathcal{C}_{1}$. By Lemma A.9, a type- $\underline{v}$ consumer also accepts all contracts in $\mathcal{C}_{0}$. Hence, a type- $\underline{v}$ consumer does search in equilibrium.

Suppose $u(\hat{\sigma} \mid \underline{v})=0$. Then the profits of a firm offering $\hat{\sigma}$ are at most $(1-\gamma)(\underline{v}-c)-\phi$. Noting Assumption A-1, such a firm can strictly increase its profit by offering the contract $\langle 0, z\rangle$ instead since $(1-\gamma)(\underline{v}-c)-\phi \leq(1-\gamma)(\underline{v}-c-\phi) \leq(1-\gamma) z$. Hence, $u(\hat{\sigma} \mid \underline{v})=u^{*}(\underline{v})>0$.

Next, suppose there exists $\tilde{\sigma} \in \mathcal{C}_{0}$ such that $u(\tilde{\sigma} \mid \bar{v})>u^{*}(\bar{v})$. Since $u^{*}(\bar{v}) \geq u^{*}(\underline{v})$, $u(\tilde{\sigma} \mid \bar{v})=u(\tilde{\sigma} \mid \underline{v})>u^{*}(\underline{v})$. This, however, contradicts Lemma A.2. Therefore, $u(\sigma \mid \bar{v}) \leq u^{*}(\bar{v})$ for all $\sigma \in \mathcal{C}_{0}$. Thus, noting Lemma A.3, we conclude that there exists $\check{\sigma} \in \mathcal{C}_{1}$ such that $u(\check{\sigma} \mid \bar{v})>u^{*}(\bar{v}) \geq u^{*}(\underline{v}) \geq 0$. But this also contradicts Lemma A.2.

Lemma A.12. $\left|\mathcal{C}_{1}\right| \leq 2$.

Proof. Let $\sigma=\langle 1, p\rangle$ and $\hat{\sigma}=\langle 1, \hat{p}\rangle$ and suppose $\sigma, \hat{\sigma} \in \mathcal{C}_{1}$. Without loss of generality, suppose $0<p<\hat{p} \leq \bar{v}$. Moreover, suppose $\sigma$ is the least-expensive contract in $\mathcal{C}_{1}$. By Lemma A.11, both contracts are accepted by type- $\bar{v}$ consumers:

$$
u(\sigma \mid \bar{v})>u(\hat{\sigma} \mid \bar{v}) \geq \max \left\{u^{*}(\bar{v}), 0\right\} .
$$


If $u(\sigma \mid \underline{v})<\max \left\{u^{*}(\underline{v}), 0\right\}$, Lemma A.2 is violated. Thus,

$$
u(\sigma \mid \underline{v}) \geq \max \left\{u^{*}(\underline{v}), 0\right\} .
$$

If the inequality in (A.5) was strict, Lemma A.2 would be violated. Thus, $u(\sigma \mid \underline{v})=$ $\max \left\{u^{*}(\underline{v}), 0\right\}>u(\hat{\sigma} \mid \underline{v})$. But then Lemma A.2 implies that $u(\hat{\sigma} \mid \bar{v})=\max \left\{u^{*}(\bar{v}), 0\right\}$. Since $\hat{\sigma}$ was an arbitrary contract in $\mathcal{C}_{1} \backslash\{\sigma\}$, we conclude that there are at most two distinct elements in $\mathcal{C}_{1}$.

\section{A.4 Proof of Theorem 3}

Theorem 3 draws on three preliminary results: Lemma 1, which is stated in the main text, Lemma A.13, and Lemma A.14. The proof of Corollary 1 follows the proof of Theorem 3.

Proof of Lemma 1. Suppose a typical firm follows the mixed strategy $\psi^{*}$ where it selects $\sigma_{1}$ with probability $\lambda$ and $\sigma_{0}$ with probability $1-\lambda$. By Lemma A.11, $\sigma_{1}$ must be accepted by a type- $\bar{v}$ consumer: $u\left(\sigma_{1} \mid \bar{v}\right) \geq \max \left\{u^{*}(\bar{v}), 0\right\}$. By Lemma A.7, $\sigma_{1}$ must also be accepted by a type- $\underline{v}$ consumer: $u\left(\sigma_{1} \mid \underline{v}\right) \geq \max \left\{u^{*}(\underline{v}), 0\right\}$. By Lemma A.9, $\sigma_{0}$ must be accepted by a type- $\underline{v}$ consumer: $u\left(\sigma_{0} \mid \underline{v}\right) \geq \max \left\{u^{*}(\underline{v}), 0\right\}$.

Suppose $u\left(\sigma_{1} \mid \underline{v}\right)>\max \left\{u^{*}(\underline{v}), 0\right\}$. Then, $u\left(\sigma_{1} \mid \bar{v}\right)=\max \left\{u^{*}(\bar{v}), 0\right\}$, else Lemma A.2 is violated. Since $u\left(\sigma_{1} \mid \bar{v}\right)>0, u^{*}(\bar{v})>0$. By Lemma A.3, $u\left(\sigma_{0} \mid \bar{v}\right)>u^{*}(\bar{v})$, which implies $u\left(\sigma_{0} \mid \underline{v}\right)>\max \left\{u^{*}(\underline{v}), 0\right\}$. However, this conclusion contradicts Lemma A.2. Therefore, $u\left(\sigma_{1} \mid \underline{v}\right)=\max \left\{u^{*}(\underline{v}), 0\right\}$. Now there are two cases.

1. Suppose $u\left(\sigma_{0} \mid \underline{v}\right)>\max \left\{u^{*}(\underline{v}), 0\right\}$. Then if $u\left(\sigma_{0} \mid \bar{v}\right) \neq \max \left\{u^{*}(\bar{v}), 0\right\}$, Lemma A.2 is contradicted. Hence, $u\left(\sigma_{0} \mid \bar{v}\right)=\max \left\{u^{*}(\bar{v}), 0\right\}$ and so $u\left(\sigma_{1} \mid \bar{v}\right)>\max \left\{u^{*}(\bar{v}), 0\right\}$ by Lemma A.3. Therefore, on the equilibrium path no consumers search and the profit of a typical firm is $p_{0}=p_{1}-c-\phi$.

Suppose a firm offered the contract $\tilde{\sigma}_{0}=\left\langle 0, \tilde{p}_{0}\right\rangle$ where $\tilde{p}_{0}=p_{1}-\underline{v}+z$. Since $u\left(\tilde{\sigma}_{0} \mid \underline{v}\right)=$ $z-\tilde{p}_{0}=\underline{v}-p_{1}=u\left(\sigma_{1} \mid \underline{v}\right)$, this is a feasible contract and it is accepted by all type- $\underline{v}$ consumers. Therefore, it gives the firm profits of at least $(1-\gamma) \tilde{p}_{0}$. However, from Assumption A-1 and the fact that $p_{1} \leq \underline{v}$ we conclude that

$$
\begin{aligned}
(1-\gamma) z>\underline{v}-c-\phi & \Longleftrightarrow(1-\gamma) z>(1-\gamma) \underline{v}+\gamma p_{1}-c-\phi \\
& \Longleftrightarrow(1-\gamma)(z-\underline{v})+(1-\gamma) p_{1}>(1-\gamma) p_{1}+\gamma p_{1}-c-\phi \\
& \Longleftrightarrow(1-\gamma)\left(p_{1}+z-\underline{v}\right)>p_{1}-c-\phi \\
& \Longleftrightarrow(1-\gamma) \tilde{p}_{0}>p_{1}-c-\phi
\end{aligned}
$$

Hence, a firm has a profitable deviation in offering $\tilde{\sigma}$. Hence, $u\left(\sigma_{0} \mid \underline{v}\right)>\max \left\{u^{*}(\underline{v}), 0\right\}$ is not compatible with an equilibrium.

2. Suppose $u\left(\sigma_{0} \mid \underline{v}\right)=\max \left\{u^{*}(\underline{v}), 0\right\}$. Then $u\left(\sigma_{1} \mid \underline{v}\right)=u\left(\sigma_{0} \mid \underline{v}\right)$. Hence, $u^{*}(\underline{v}) \leq 0$ and $0=u\left(\sigma_{1} \mid \underline{v}\right)=u\left(\sigma_{0} \mid \underline{v}\right)$. But this implies $p_{1}=\underline{v}$ and $p_{0}=z$, as required. 
Lemma A.13. Suppose all firms are following the strategy $\psi^{*}$ where a firm selects $\sigma_{1}$ with probability $\lambda$ and $\sigma_{0}$ with probability $1-\lambda$. Suppose all consumers adopt an optimal search strategy holding correct beliefs, $\tilde{\psi}=\psi^{*}$. Suppose $u\left(\sigma_{0} \mid \underline{v}\right) \geq \max \left\{u^{*}(\underline{v}), 0\right\}$, $u\left(\sigma_{1} \mid \underline{v}\right) \geq \max \left\{u^{*}(\underline{v}), 0\right\}$, and $u\left(\sigma_{1} \mid \bar{v}\right) \geq \max \left\{u^{*}(\bar{v}), 0\right\}>u\left(\sigma_{0} \mid \bar{v}\right)$. Suppose firm $j$ deviates from $\psi^{*}$ and offers $\sigma_{j}$ such that $u\left(\sigma_{j} \mid \bar{v}\right) \geq \max \left\{u^{*}(\bar{v}), 0\right\}$. Then the expected number of consumers with indices $i<j$ who accept firm $j$ 's contract is $\gamma\left(\frac{1}{\lambda}-1\right)$.

Proof. Given the contracts available, type- $\underline{v}$ consumers do not search. Type- $\bar{v}$ consumers search when they match to $\sigma_{0}$. Otherwise, they accept $\sigma_{1}$ or $\sigma_{j}$ should they reach firm $j$.

First, we calculate the probability that exactly $q$ consumers with indices $i<j$ accept firm $j$ 's contract. Let $k \geq q$. Conditional on all firms $j^{\prime} \in\{j-k, \ldots, j-1\}$ offering the contract $\sigma_{0}$ and firm $j-k-1$ offering the contract $\sigma_{1}$, for $q$ consumers with indices $i<j$ to accept the contract offered by firm $j$, exactly $q$ consumers with indices $j-k \leq i \leq j-1$ must be of type- $\bar{v}$. In this case, no consumers with indices $i \leq j-k-1$ will search long enough to become acquainted with the contract offer of firm $j$. Thus, conditional on such a contract offer by firms, the probability that $q$ consumers with indices $i<j$ accept the contract offered by firm $j$ is $\left(\begin{array}{l}k \\ q\end{array}\right) \gamma^{q}(1-\gamma)^{k-q}$. For each $k$, the events described above are disjoint. Thus, the probability that exactly $q$ consumers with indices $i<j$ accept firm $j$ 's contract is

$$
\sum_{k=q}^{\infty} \lambda(1-\lambda)^{k}\left[\left(\begin{array}{l}
k \\
q
\end{array}\right) \gamma^{q}(1-\gamma)^{k-q}\right]=(1-\lambda)^{q} \lambda \gamma^{q}(\lambda+\gamma-\gamma \lambda)^{-1-q}
$$

We can use this probability to compute the expected number of consumers with indices $i<j$ accepting $j$ 's contract offer. This calculation simplifies conveniently:

$\sum_{q=1}^{\infty} q \cdot\left[(1-\lambda)^{q} \lambda \gamma^{q}(\lambda+\gamma-\gamma \lambda)^{-1-q}\right]=\gamma\left(\frac{1}{\lambda}-1\right)$.

Lemma A.14. There exists a unique $\lambda^{*} \in(0,1)$ such that $\left(1-\gamma+\frac{\gamma}{\lambda^{*}}\right)(\underline{v}-c)-\phi=(1-\gamma) z$. In fact, $\lambda^{*}=\frac{\gamma(\underline{v}-c)}{\phi-(1-\gamma)(\underline{v}-c-z)}$.

Proof. The function $f(\lambda)=\left(1-\gamma+\frac{\gamma}{\lambda}\right)(\underline{v}-c)-\phi$ is continuous on $(0,1]$ and strictly decreasing. Moreover, $\lim _{\lambda \rightarrow 0^{+}} f(\lambda)=\infty$ and $f(1)=\underline{v}-c-\phi<(1-\gamma) z$. Hence, there exists a unique $\lambda^{*}$ such that $f\left(\lambda^{*}\right)=(1-\gamma) z$. Solving this equation gives $\lambda^{*}=\frac{\gamma(\underline{v}-c)}{\phi-(1-\gamma)(\underline{v}-c-z)}$.

Proof of Theorem 3. Suppose a typical firm follows the mixed strategy $\psi^{*}$ where it selects $\sigma_{1}$ with probability $\lambda_{1}^{*}$ and $\sigma_{0}$ with probability $1-\lambda_{1}^{*}$. When a consumer's beliefs are that $\tilde{\psi}=\psi^{*}$, his search strategy is defined by the cutoff values $u^{*}(\underline{v})=-s$ and $u^{*}(\bar{v})=\bar{v}-\underline{v}-s / \lambda_{1}^{*}$. Condition (2) implies $u^{*}(\bar{v})=\bar{v}-\underline{v}-s / \lambda_{1}^{*} \geq 0 .^{2}$ Hence, a type- $\bar{v}$ consumer will search for $\sigma_{1}$ in lieu of accepting $\sigma_{0}$ or opting out of the market. He will stop his search once he encounters a contract $\tilde{\sigma}$ such that $u(\tilde{\sigma} \mid \bar{v}) \geq u^{*}(\underline{v})$. A type- $\underline{v}$ consumer does not search.

\footnotetext{
${ }^{2}$ To confirm this fact, it is sufficient to verify that $z-c-\phi /\left(1-\gamma+\gamma / \lambda_{1}^{*}\right) \geq \underline{v}$. If we suppose the contrary and substitute for $\lambda_{1}^{*}$ we observe that $\underline{v}>z+c+\phi(\underline{v}-c) /(\phi+z(1-\gamma))>z+c+\underline{v}-c$, which is a contradiction.
} 
We next compute a firm's expected profits. If firm $j$ offers $\tilde{\sigma}$, which is acceptable to both types of consumers, the expected total number of consumers who accept firm $j$ 's offer is $1-\gamma+\frac{\gamma}{\lambda_{1}^{*}}$. Consumer $i=j$ accepts the contract without engaging in search. To this one consumer, we add $\gamma / \lambda_{1}^{*}-\gamma$ (Lemma A.13). This is the expected number of type- $\bar{v}$ consumers who end up accepting firm $j$ 's contract offer. In particular, when firm $j$ offers $\sigma_{1}$, its expected profits are $\Pi_{j}\left(\sigma_{1}\right)=\left(1+\frac{\gamma}{\lambda_{1}^{*}}-\gamma\right)(\underline{v}-c)-\phi$. If firm $j$ offers $\sigma_{0}$, its expected profits are $\Pi_{j}\left(\sigma_{0}\right)=(1-\gamma) z$. An equilibrium requires $\Pi_{j}\left(\sigma_{0}\right)=\Pi_{j}\left(\sigma_{1}\right)$. By Lemma A.14, there exists a unique $\lambda_{1}^{*} \in(0,1)$ ensuring this equality: $\lambda_{1}^{*}=\frac{\gamma(\underline{v}-c)}{\phi-(1-\gamma)(\underline{v}-c-z)}$. Thus, both $\sigma_{0}$ and $\sigma_{1}$ give a firm the same expected profits.

The final step is to verify that no firm can profitably deviate. There are two relevant classes of deviations. A firm may offer good 0 at a price less than $z$ or good 1 at a price more than $\underline{v}$. Other deviations are clearly not optimal.

1. Suppose firm $j$ offers $\tilde{\sigma}=\langle 0, \tilde{p}\rangle, \tilde{p}<z$. If $\tilde{p}$ is very close to $z$, the firm's expected profits will decline. For $\tilde{\sigma}$ to increase the firm's profits, it must entice type- $\bar{v}$ consumers who otherwise would search to stop and to accept it. Thus, $z-\tilde{p} \geq \bar{v}-\underline{v}-s / \lambda_{1}^{*}$. To maximize its expected profit from this deviation, the firm would set $\tilde{p}=z-\bar{v}+\underline{v}+$ $s / \lambda_{1}^{*}$. Hence, the maximal expected profits associated with this alternative strategy are $\Pi_{j}(\tilde{\sigma})=\left(1+\gamma / \lambda_{1}^{*}-\gamma\right)\left(z-\bar{v}+\underline{v}+s / \lambda_{1}^{*}\right)$. Rearranging condition (2) we conclude that $\Pi_{j}\left(\sigma_{1}\right) \geq \Pi_{j}(\tilde{\sigma})$. Hence, firm $j$ cannot increase its profits through this deviation.

2. Suppose firm $j$ offers $\tilde{\sigma}=\langle 1, \tilde{p}\rangle$ where $\tilde{p}>\underline{v}$. In this case, no type- $\underline{v}$ consumers will accept the contract. A type- $\bar{v}$ consumer will accept $\tilde{\sigma}$ if and only if $\bar{v}-\tilde{p} \geq \bar{v}-\underline{v}-s / \lambda_{1}^{*}$. The maximum price which will be accepted is $\tilde{p}=\underline{v}+s / \lambda_{1}^{*}$. When $\tilde{p}=\underline{v}+s / \lambda_{1}^{*}$, the expected profit is $\Pi_{j}(\tilde{\sigma})=\left(\gamma+\frac{\gamma}{\lambda_{1}^{*}}-\gamma\right)(\tilde{p}-c)-\phi=\frac{\gamma}{\lambda_{1}^{*}}\left(\underline{v}-c+\frac{s}{\lambda_{1}^{*}}\right)-\phi$. However, $\Pi_{j}(\tilde{\sigma})-\Pi_{j}\left(\sigma_{1}\right)=\frac{\gamma s}{\left(\lambda_{1}^{*}\right)^{2}}-(1-\gamma)(\underline{v}-c) \leq 0$. Where the inequality follows from condition $(3)$.

Proof of Corollary 1. From Theorem 3 it follows that if $\psi^{*}$ is the equilibrium strategy of a firm when the search costs are $\bar{s}, \psi^{*}$ continues to be an equilibrium if search costs are $s \in(0, \bar{s})$.

The expected profit of a typical firm in the equilibrium of Theorem 3 is $(1-\gamma) z$. All type- $\underline{v}$ consumers expect a payoff of zero. Type- $\bar{v}$ consumers earn an expected payoff of $\lambda_{1}^{*}(\bar{v}-\underline{v})+\left(1-\lambda_{1}^{*}\right)\left(\bar{v}-\underline{v}-s / \lambda_{1}^{*}\right)$. Hence, total expected surplus for a firm-consumer pair is $(1-\gamma) z+\gamma\left(\lambda_{1}^{*}(\bar{v}-\underline{v})+\left(1-\lambda_{1}^{*}\right)\left(\bar{v}-\underline{v}-s / \lambda_{1}^{*}\right)\right)$. This expression must exceed $z$. Taking the limit $s \rightarrow 0^{+}$, we see that such an outcome is possible if and only if $\bar{v}-\underline{v} \geq z$.

\section{A.5 Multiple Goods Trading at Multiple Prices}

Proof of Lemma 2. The result follows from Lemmas A.5-A.12 in the proof of Theorem 2. 
1. It is sufficient to establish that $u\left(\sigma_{0} \mid \underline{v}\right)>u\left(\hat{\sigma}_{0} \mid \underline{v}\right)=\max \left\{u^{*}(\underline{v}), 0\right\}$ and $u\left(\sigma_{0} \mid \bar{v}\right)=u^{*}(\bar{v})$. By Lemma A.9, $\sigma_{0}$ and $\hat{\sigma}_{0}$ must be acceptable to a type- $\underline{v}$ consumer. Thus, $u\left(\sigma_{0} \mid \underline{v}\right)>$ $u\left(\hat{\sigma}_{0} \mid \underline{v}\right)=\max \left\{u^{*}(\underline{v}), 0\right\}$. The equality is due to (A.2) in the proof of Lemma A.10. From (A.3) we have $u\left(\sigma_{0} \mid \bar{v}\right)=u^{*}(\bar{v})$ as needed.

2. It is sufficient to establish that $u\left(\sigma_{1} \mid \underline{v}\right)=\max \left\{u^{*}(\underline{v}), 0\right\}$ and $u\left(\sigma_{1} \mid \bar{v}\right)>u^{*}(\bar{v})=u\left(\hat{\sigma}_{1} \mid \bar{v}\right)$. By Lemma A.11, $\sigma_{1}$ and $\hat{\sigma}_{1}$ must be acceptable to a type- $\bar{v}$ consumer. Thus, $u\left(\sigma_{1} \mid \bar{v}\right)>$ $u\left(\hat{\sigma}_{1} \mid \bar{v}\right)=u^{*}(\bar{v})$ where the equality follows from the proof of Lemma A.12. Also from the proof of Lemma A.12 we have $u\left(\sigma_{1} \mid \underline{v}\right)=\max \left\{u^{*}(\underline{v}), 0\right\}$.

Proof of Lemma 3. By Theorem 2, only four classes of equilibria may exist in our economy. We analyzing the case of product differentiation and no price dispersion, we showed that a typical firm's profits are $(1-\gamma) z$. Therefore, if a Pareto improvement is to occur at least one good must trade at multiple prices.

1. Suppose there is an equilibrium where firms offer only $\sigma_{0}, \sigma_{1}$, and $\hat{\sigma}_{0}$ with positive probability. That is, only good 0 trades at multiple prices. From Lemma 2 we know that $u\left(\sigma_{0} \mid \underline{v}\right)>u\left(\hat{\sigma}_{0} \mid \underline{v}\right)=\max \left\{u^{*}(\underline{v}), 0\right\}$ and $u\left(\sigma_{0} \mid \bar{v}\right)=u^{*}(\bar{v})>u\left(\hat{\sigma}_{0} \mid \bar{v}\right)$. Hence $\hat{\sigma}_{0}$ is acceptable only to type- $\underline{v}$ consumers and is not accepted by type- $\bar{v}$ consumers. Moreover, type- $\underline{v}$ consumers always accept $\sigma_{0}$.

Suppose that type- $\underline{v}$ consumers always accept $\sigma_{1}$. Then the expected profit of a firm offering $\hat{\sigma}_{0}$ is at most $(1-\gamma) z$, which is not reflective of a Pareto improvement. Therefore, a type- $\underline{v}$ consumer must search with positive probability if his only available contract is $\sigma_{1}$. That is, $u\left(\sigma_{1} \mid \underline{v}\right) \leq u^{*}(\underline{v})$. There are two relevant sub cases:

(a) Suppose $u\left(\sigma_{1} \mid \underline{v}\right)<u^{*}(\underline{v})$. Therefore, only type- $\bar{v}$ consumers accept $\sigma_{1}$. If $u\left(\sigma_{1} \mid \bar{v}\right)=$ $\bar{v}-p_{1}>\max \left\{u^{*}(\bar{v}), 0\right\}$, then a typical firm offering $\sigma_{1}$ could slightly increase its price without affecting its demand. Therefore, in equilibrium the price of good 1 must satisfy $\bar{v}-p_{1}=\max \left\{u^{*}(\bar{v}), 0\right\}$. But this leads to a contradiction. Specifically, if $\bar{v}-p_{1}=u\left(\sigma_{1} \mid \bar{v}\right)=u^{*}(\bar{v}) \geq 0$, then using Lemmas A.1 and 2 we see that $u^{*}(\bar{v})=\lambda_{0} u^{*}(\bar{v})+\hat{\lambda}_{0} u^{*}(\bar{v})+\lambda_{1} \max \left\{u^{*}(\bar{v}), 0\right\}-s$, which is impossible. On the other hand, if $u^{*}(\bar{v})<0$ then the profits generated by the contract $\sigma_{1}$ are at most $\gamma(\bar{v}-c)-\phi$, which is less than $z$ by Assumption A-1. Hence, this outcome cannot lead to a Pareto improvement.

(b) Suppose $u\left(\sigma_{1} \mid \underline{v}\right)=u^{*}(\underline{v})$. In this case, a type- $\underline{v}$ consumer is indifferent between searching for an alternative offer and accepting $\sigma_{1}$. Any firm offering $\sigma_{1}$ can slightly decrease its price (which is strictly positive) and discretely increase its expected profits by encouraging type- $\underline{v}$ consumers to accept its offer rather than search. Hence, this case is not compatible with an equilibrium.

2. Suppose there is an equilibrium where firms offer only $\sigma_{0}, \sigma_{1}$, and $\hat{\sigma}_{1}$ with positive probability. That is, only good 1 trades at multiple prices. From Lemma 2 we know 
that $u\left(\sigma_{1} \mid \underline{v}\right)=\max \left\{u^{*}(\underline{v}), 0\right\}>u\left(\hat{\sigma}_{1} \mid \underline{v}\right)$ and $u\left(\sigma_{1} \mid \bar{v}\right)>u\left(\hat{\sigma}_{1} \mid \bar{v}\right)=u^{*}(\bar{v})$. Therefore, $\hat{\sigma}_{1}$ is only acceptable to type- $\bar{v}$ consumers. If all type- $\bar{v}$ consumers accept $\sigma_{0}$, then the expected profits of a typical firm offering $\hat{\sigma}_{1}$ are at most $\gamma(\bar{v}-c)-\phi$, which is less than $z$ by Assumption A-1. Hence, some type- $\bar{v}$ consumers must search if $\sigma_{0}$ is their only available contract. That is, $u\left(\sigma_{0} \mid \bar{v}\right) \leq u^{*}(\bar{v})$. There are two sub cases to consider.

(a) If $u\left(\sigma_{0} \mid \bar{v}\right)<u^{*}(\bar{v})$, then no type- $\bar{v}$ consumers accept $\sigma_{0}$. For $\sigma_{0}$ to generate an expected profit greater than $z, u\left(\sigma_{0} \mid \underline{v}\right)>\max \left\{u^{*}(\underline{v}), 0\right\}$, so as to entice some type- $\underline{v}$ consumers to search. (The strict inequality is necessary.) However, this implies that any firm offering $\sigma_{0}$ can strictly increase its price slightly without affecting its demand, contradicting the equilibrium condition.

(b) If $u\left(\sigma_{0} \mid \bar{v}\right)=u^{*}(\bar{v})$, then any firm offering $\sigma_{0}$ can strictly increase its profit by reducing $p_{0}$ slightly thereby encouraging type- $\bar{v}$ consumers to accept $\sigma_{0}$ in lieu of searching for a good 1 contract. This contradicts the equilibrium condition.

Hence, three of the four possible classes of equilibria are not compatible with a Paretoimproving outcome. Therefore, if there is an equilibrium with the desired characteristics, it must involve two goods trading at multiple prices.

Second, the requisite pattern of consumer search follows from Lemma 2. First, $u^{*}(\bar{v})>0$ since $u^{*}(\bar{v})=u\left(\sigma_{0} \mid \bar{v}\right)=z-p_{0}>z-\hat{p}_{0}=u(\hat{\sigma} \mid \underline{v})=\max \left\{u^{*}(\bar{v}), 0\right\} \geq 0$. Second, if $u^{*}(\underline{v})<0$, then $\hat{\sigma}_{0}$ is acceptable only to a type- $\underline{v}$ consumer. But this implies that $\left.\Pi\left(\hat{\sigma}_{0}\right)\right)=(1-\gamma) z$. Thus, firms' equilibrium profits fall below the benchmark case.

Remark A.1. In any equilibrium where a consumer is indifferent between accepting a contract and continued search, he must accept the contract. Else, a firm offering the contract in question can lower the price slightly prompting a discrete increase in expected profits.

Proof of Lemma 4. Since $u^{*}(\bar{v}) \geq u^{*}(\underline{v}) \geq 0$, Lemma 2 implies that $z-p_{0}=u^{*}(\bar{v})>z-\hat{p}_{0}$ and $\bar{v}-p_{1}>\bar{v}-\hat{p}_{1}=u^{*}(\bar{v})$. We can write $(\mathrm{A} .1)$ as

$$
\begin{aligned}
u^{*}(\bar{v}) & =\lambda_{0}\left(z-p_{0}\right)+\hat{\lambda}_{0} \max \left\{z-\hat{p}_{0}, 0, u^{*}(\bar{v})\right\}+\lambda_{1}\left(\bar{v}-p_{1}\right)+\hat{\lambda}_{1}\left(\bar{v}-\hat{p}_{1}\right)-s \\
& =\lambda_{0} u^{*}(\bar{v})+\hat{\lambda}_{0} u^{*}(\bar{v})+\lambda_{1}\left(\bar{v}-p_{1}\right)+\hat{\lambda}_{1} u^{*}(\bar{v})-s \\
& =\left(1-\lambda_{1}\right) u^{*}(\bar{v})+\lambda_{1}\left(\bar{v}-p_{1}\right)-s \\
\Longleftrightarrow u^{*}(\bar{v}) & =\bar{v}-p_{1}-s / \lambda_{1}
\end{aligned}
$$

The argument for $u^{*}(\underline{v})$ is analogous.

To derive the relationships among prices we use equalities from Lemma 2 and the imme- 
diately preceding computations.

$$
\begin{aligned}
& u\left(\hat{\sigma}_{0} \mid \underline{v}\right)=u^{*}(\underline{v}) \Longrightarrow z-\hat{p}_{0}=z-p_{0}-\frac{s}{\lambda_{0}} \Longrightarrow \hat{p}_{0}=p_{0}+\frac{s}{\lambda_{0}} \\
& u\left(\sigma_{1} \mid \underline{v}\right)=u^{*}(\underline{v}) \Longrightarrow \underline{v}-p_{1}=z-p_{0}-\frac{s}{\lambda_{0}} \Longrightarrow p_{1}=p_{0}+\frac{s}{\lambda_{0}}+\underline{v}-z \\
& u\left(\sigma_{0} \mid \bar{v}\right)=u^{*}(\bar{v}) \Longrightarrow z-p_{0}=\bar{v}-p_{1}-\frac{s}{\lambda_{1}} \Longrightarrow p_{0}=p_{1}+\frac{s}{\lambda_{1}}+z-\bar{v} \\
& u\left(\hat{\sigma}_{1} \mid \bar{v}\right)=u^{*}(\bar{v}) \Longrightarrow \bar{v}-\hat{p}_{1}=\bar{v}-p_{1}-\frac{s}{\lambda_{1}} \Longrightarrow \hat{p}_{1}=p_{1}+\frac{s}{\lambda_{1}}
\end{aligned}
$$

Proof of Lemma 5. From Section IV.B, we can easily describe consumer search behavior. A type- $\underline{v}$ consumer searches for an alternative offer if $\hat{\sigma}_{1}$ is the only contract that is available. He accepts $\sigma_{0}, \hat{\sigma}_{0}$, and $\sigma_{1}$. A type- $\bar{v}$ consumer searches for an alternative offer if $\hat{\sigma}_{0}$ is the only contract that is available. He accepts $\sigma_{0}, \sigma_{1}$, and $\hat{\sigma}_{1}$.

1. The contract $\sigma_{0}=\left\langle 0, p_{0}\right\rangle$ is acceptable to both types of consumers. We must compute the expected number of consumers with indices $i<j$ who accept firm $j$ 's offer.

Suppose for the moment that at least one type- $\bar{v}$ consumer with an index $i<j$ accepts $\sigma_{0}$ from firm $j$. This implies that firm $j-1$ must be offering the contract $\hat{\sigma}_{0}=\left\langle 0, \hat{p}_{0}\right\rangle$. But then no type- $\underline{v}$ consumers with indices $i<j$ will accept firm $j$ 's offer. Any type- $\underline{v}$ consumer who is searching would accept $\hat{\sigma}_{0}$ from firm $j-1$ before learning the offer of firm $j$. Likewise, if at least one type- $\underline{v}$ consumer with an index $i<j$ accepts $\sigma_{0}$ from firm $j$, then no type- $\bar{v}$ consumers with indices $i<j$ will accept the offer from firm $j$. Thus, firm $j$ sells its product to either $q$ consumers of type- $\bar{v}$ who are searching or to $q^{\prime}$ consumers of type- $\underline{v}$ who are searching. Never does it sell its product to a mixed group of buyers with indices $i<j$.

Following the reasoning in the proof of Lemma A.13, the probability that exactly $q$ type- $\bar{v}$ consumers who are searching accept firm $j$ 's offer is $\sum_{k=q}^{\infty} \hat{\lambda}_{0}^{k}\left(1-\hat{\lambda}_{0}\right)\left(\begin{array}{l}k \\ q\end{array}\right) \gamma^{q}(1-$ $\gamma)^{k-q}=\frac{\left(1-\hat{\lambda}_{0}\right) \hat{\lambda}_{0}^{q} \gamma^{q}}{\left(1-\hat{\lambda}_{0}(1-\gamma)\right)^{q+1}}$. Similarly, the probability that exactly $q$ type- $\underline{v}$ consumers who are searching accept firm $j$ 's offer is $\sum_{k=q}^{\infty} \hat{\lambda}_{1}^{k}\left(1-\hat{\lambda}_{1}\right)\left(\begin{array}{l}k \\ q\end{array}\right)(1-\gamma)^{q} \gamma^{k-q}=\frac{\left(1-\hat{\lambda}_{1}\right) \hat{\lambda}_{1}^{q}(1-\gamma)^{q}}{\left(1-\hat{\lambda}_{1} \gamma\right)^{q+1}}$. We can compute the expected number of consumers with indices $i<j$ who accept firm $j$ 's offer of $\sigma_{0}$ to be

$$
\sum_{q=1}^{\infty}\left[\frac{\left(1-\hat{\lambda}_{0}\right) \hat{\lambda}_{0}^{q} \gamma^{q}}{\left(1-\hat{\lambda}_{0}(1-\gamma)\right)^{q+1}}+\frac{\left(1-\hat{\lambda}_{1}\right) \hat{\lambda}_{1}^{q}(1-\gamma)^{q}}{\left(1-\hat{\lambda}_{1} \gamma\right)^{q+1}}\right] q=\frac{\gamma \hat{\lambda}_{0}}{1-\hat{\lambda}_{0}}+\frac{(1-\gamma) \hat{\lambda}_{1}}{1-\hat{\lambda}_{1}}
$$

Hence, accounting for consumer $i=j$ who also accepts $\sigma_{0}$, the expected profit of firm $j$ is $\Pi_{j}\left(\sigma_{0}\right)=\left(1+\frac{\gamma \hat{\lambda}_{0}}{1-\hat{\lambda}_{0}}+\frac{(1-\gamma) \hat{\lambda}_{1}}{1-\hat{\lambda}_{1}}\right) p_{0}=\left(\frac{\gamma}{1-\hat{\lambda}_{0}}+\frac{1-\gamma}{1-\hat{\lambda}_{1}}\right) p_{0}$.

2. The reasoning is identical to the preceding case. 
3. The contract $\hat{\sigma}_{0}=\left\langle 0, \hat{p}_{0}\right\rangle$ is accepted only by type- $\underline{v}$ consumers. Therefore, noting the above calculations, the expected number of type- $\underline{v}$ consumers who accept $\left\langle 0, \hat{p}_{0}\right\rangle$ is $\left[(1-\gamma)+\frac{(1-\gamma) \hat{\lambda}_{1}}{1-\hat{\lambda}_{1}}\right]=\frac{1-\gamma}{1-\hat{\lambda}_{1}}$. Hence, the expected profit is $\Pi_{j}\left(\hat{\sigma}_{0}\right)=\frac{1-\gamma}{1-\hat{\lambda}_{1}} \hat{p}_{0}$.

4. The reasoning is identical to the preceding case.

Lemma A.15. There exists an equilibrium where both goods trade at multiple prices and consumer search conforms to the pattern described in Lemma 3 if and only if there exists strictly positive constants $-\lambda_{0}, \lambda_{1}, \hat{\lambda}_{0}, \hat{\lambda}_{1}$-such that

$$
\begin{aligned}
\bar{v}-\underline{v} & =\frac{s}{\lambda_{0}}+\frac{s}{\lambda_{1}} \\
\Pi_{j}\left(\sigma_{0}\right) & =\Pi_{j}\left(\sigma_{1}\right) \\
p_{1} & =p_{0}+\frac{s}{\lambda_{0}}+\underline{v}-z \\
1 & =\lambda_{0}+\lambda_{1}+\hat{\lambda}_{0}+\hat{\lambda}_{1} \\
z-p_{0}-\frac{s}{\lambda_{0}} & \geq 0
\end{aligned}
$$

where

$$
p_{0}=\frac{1-\hat{\lambda}_{0}}{1-\hat{\lambda}_{1}} \cdot \frac{1-\gamma}{\gamma} \cdot \frac{s}{\lambda_{0}} \quad \text { and } \quad p_{1}=c+\frac{1-\hat{\lambda}_{1}}{1-\hat{\lambda}_{0}} \cdot \frac{\gamma}{1-\gamma} \cdot \frac{s}{\lambda_{1}} .
$$

Proof. Conditions (A.6)-(A.10) reproduce the conditions identified in Section IV.B. Thus, necessity follows from the discussion in that section. To prove sufficiency we reverse the argument. Let $\lambda_{0}, \lambda_{1}, \hat{\lambda}_{0}$, and $\hat{\lambda}_{1}$ be the strictly positive values that satisfy the conditions (A.6)-(A.10). We use these values to define four contracts, two for each good, as follows:

- Let $\sigma_{0}=\left\langle 0, p_{0}\right\rangle$ be a contract supplying good 0 at a price of

$$
p_{0}=\frac{1-\hat{\lambda}_{0}}{1-\hat{\lambda}_{1}} \cdot \frac{1-\gamma}{\gamma} \cdot \frac{s}{\lambda_{0}} .
$$

- Let $\hat{\sigma}_{0}=\left\langle 0, \hat{p}_{0}\right\rangle$ be a contract supplying good 0 at a price of $\hat{p}_{0}=p_{0}+s / \lambda_{0}$, where $p_{0}$ is given by (A.12).

- Let $\sigma_{1}=\left\langle 1, p_{1}\right\rangle$ be a contract supplying good 1 at a price of

$$
p_{1}=c+\frac{1-\hat{\lambda}_{1}}{1-\hat{\lambda}_{0}} \cdot \frac{\gamma}{1-\gamma} \cdot \frac{s}{\lambda_{1}} .
$$

- Let $\hat{\sigma}_{1}=\left\langle 1, \hat{p}_{1}\right\rangle$ be a contract supplying good 1 at a price of $\hat{p}_{1}=p_{1}+s / \lambda_{1}$, where $p_{1}$ is given by (A.13). 
Suppose each firm adopts the strategy $\psi_{j}^{*}$ where contract $\sigma_{k}\left(\hat{\sigma}_{k}\right)$ is offered with probability $\lambda_{k}\left(\hat{\lambda}_{k}\right)$. Since $1=\lambda_{0}+\lambda_{1}+\hat{\lambda}_{0}+\hat{\lambda}_{1}$, all prices defined above are strictly positive and the strategy is a valid mixed strategy. To verify the equilibrium, we first characterize consumers' search behavior given $\psi_{j}^{*}$. Subsequently we compute the expected profits associated with each contract given the consumers' search strategy. Finally, we verify that no firm has a profitable deviation from the mixed strategy $\psi_{j}^{*}$.

The Optimal Search Strategy for a Type- $\underline{\underline{v}}$ Consumer. Given the contracts defined above,

$$
\begin{array}{ll}
u\left(\sigma_{0} \mid \underline{v}\right)=z-p_{0} & u\left(\hat{\sigma}_{0} \mid \underline{v}\right)=z-\hat{p}_{0}=z-p_{0}-s / \lambda_{0} \\
u\left(\sigma_{1} \mid \underline{v}\right)=\underline{v}-p_{1} & u\left(\hat{\sigma}_{1} \mid \underline{v}\right)=\underline{v}-\hat{p}_{1}=\underline{v}-p_{1}-s / \lambda_{1} .
\end{array}
$$

(A.10) implies that $u\left(\sigma_{0} \mid \underline{v}\right)>u\left(\hat{\sigma}_{0} \mid \underline{v}\right) \geq 0$. Moreover, by (A.8), $u\left(\sigma_{1} \mid \underline{v}\right)=\underline{v}-p_{1}=\underline{v}-$ $p_{0}-s / \lambda_{0}-\underline{v}+z=z-p_{0}-s / \lambda_{0}=u\left(\hat{\sigma}_{0} \mid \underline{v}\right)$. Therefore, $u\left(\sigma_{0} \mid \underline{v}\right)>u\left(\sigma_{1} \mid \underline{v}\right)=u\left(\hat{\sigma}_{0} \mid \underline{v}\right)>u\left(\hat{\sigma}_{1} \mid \underline{v}\right)$.

If a type- $\underline{v}$ consumer believes that all firms are following the mixed strategy $\psi_{j}^{*}$, his optimal search strategy will be characterized by a constant cutoff value, $u^{*}(\underline{v})$. From Lemma A.1, $u^{*}(\underline{v})$ solves

$$
u^{*}(\underline{v})=\mathbb{E}_{\psi_{j}^{*}}\left[\max \left\{u^{*}(\underline{v}), u(\sigma \mid \underline{v}), 0\right\}\right]-s .
$$

There are two possible cases:

1. Suppose that the value $u^{*}(\underline{v})$ that solves (A.14) is such that $u\left(\sigma_{0} \mid \underline{v}\right) \geq u^{*}(\underline{v}) \geq$ $u\left(\sigma_{1} \mid \underline{v}\right)=u\left(\hat{\sigma}_{0} \mid \underline{v}\right)>u\left(\hat{\sigma}_{1} \mid \underline{v}\right)$. Then, $u^{*}(\underline{v})=\lambda_{0}\left(z-p_{0}\right)+\left(1-\lambda_{0}\right) u^{*}(\underline{v})-s \Longleftrightarrow$ $u^{*}(\underline{v})=z-p_{0}-s / \lambda_{0}$.

2. Suppose that the value $u^{*}(\underline{v})$ that solves (A.14) is such that $u\left(\sigma_{0} \mid \underline{v}\right)>u\left(\sigma_{1} \mid \underline{v}\right)=$ $u\left(\hat{\sigma}_{0} \mid \underline{v}\right)>u^{*}(\underline{v}) \geq \cdots$. Then,

$$
\begin{aligned}
u^{*}(\underline{v})= & \lambda_{0}\left(z-p_{0}\right)+\left(\hat{\lambda}_{0}+\lambda_{1}\right)\left(z-p_{0}-\frac{s}{\lambda_{0}}\right) \\
& +\hat{\lambda}_{1} \max \left\{u\left(\hat{\sigma}_{1} \mid \underline{v}\right), u^{*}(\underline{v}), 0\right\}-s \\
\Longrightarrow & u^{*}(\underline{v})=\left(1-\hat{\lambda}_{1}\right)\left(z-p_{0}\right)-\left(\hat{\lambda}_{0}+\lambda_{1}\right) \frac{s}{\lambda_{0}}+\hat{\lambda}_{1} u^{*}(\underline{v})-s \\
\Longrightarrow & u^{*}(\underline{v})=z-p_{0}-\frac{\hat{\lambda}_{0}+\lambda_{1}}{1-\hat{\lambda}_{1}} \frac{s}{\lambda_{0}}-\frac{s}{1-\hat{\lambda}_{1}}
\end{aligned}
$$

By assumption, however, $z-p_{0}-s / \lambda_{0}>u^{*}(\underline{v})$. Therefore,

$$
\frac{\hat{\lambda}_{0}+\lambda_{1}}{1-\hat{\lambda}_{1}} \frac{s}{\lambda_{0}}+\frac{s}{1-\hat{\lambda}_{1}}>\frac{s}{\lambda_{0}} \Longleftrightarrow \lambda_{0}\left(1-\hat{\lambda}_{1}\right)\left(1-\lambda_{0}-\lambda_{1}-\hat{\lambda}_{0}-\hat{\lambda}_{1}\right) s<0 .
$$

But since $1-\lambda_{0}-\lambda_{1}-\hat{\lambda}_{0}-\hat{\lambda}_{1}=0$, this final condition is a contradiction.

Since case 2 cannot apply, we conclude that under the assumed values for the $\lambda^{\prime}$ 's, $u^{*}(\underline{v})=$ $z-p_{0}-s / \lambda_{0}$ and $u^{*}(\underline{v})>u\left(\hat{\sigma}_{1} \mid \underline{v}\right)$. Thus, when a type- $\underline{v}$ consumer believes all firms adopt 
the strategy $\psi_{j}^{*}$, he finds acceptable the contracts $\sigma_{0}, \hat{\sigma}_{0}$, and $\sigma_{1}$. If $\hat{\sigma}_{1}$ is the only contract available, the consumer will search in lieu of accepting $\hat{\sigma}_{1}$ or exiting the market.

The Optimal Search Strategy for a Type- $\bar{v}$ Consumer. Given the set of contracts defined above, we can compute a type- $\bar{v}$ consumer's expected utility:

$$
\begin{array}{ll}
u\left(\sigma_{0} \mid \bar{v}\right)=z-p_{0} & u\left(\hat{\sigma}_{0} \mid \bar{v}\right)=z-\hat{p}_{0}=z-p_{0}-s / \lambda_{0} \\
u\left(\sigma_{1} \mid \bar{v}\right)=\bar{v}-p_{1} & u\left(\hat{\sigma}_{1} \mid \bar{v}\right)=\bar{v}-\hat{p}_{1}=\bar{v}-p_{1}-s / \lambda_{1}
\end{array}
$$

Again, $u\left(\sigma_{0} \mid \underline{v}\right)>u\left(\hat{\sigma}_{0} \mid \underline{v}\right) \geq 0$ and substituting the expression from above yields $u\left(\hat{\sigma}_{1} \mid \bar{v}\right)=$ $\bar{v}-p_{1}-s / \lambda_{1}=\bar{v}-p_{0}-s / \lambda_{0}-\underline{v}+z-s / \lambda_{1}=z-p_{0}$. Hence, $u\left(\sigma_{1} \mid \bar{v}\right)>u\left(\hat{\sigma}_{1} \mid \bar{v}\right)=$ $u\left(\sigma_{0} \mid \bar{v}\right)>u\left(\hat{\sigma}_{0} \mid \bar{v}\right)$. An argument analogous to the previous case establishes that under the proposed conditions, the cutoff value charactering the search strategy of a type- $\bar{v}$ consumer is $u^{*}(\bar{v})=\bar{v}-p_{1}-s / \lambda_{1}=z-p_{0}$. Thus, when a type- $\bar{v}$ consumer believes all firms adopt the strategy $\psi_{j}^{*}$, he finds acceptable the contracts $\sigma_{0}, \sigma_{1}$, and $\hat{\sigma}_{1}$. If $\hat{\sigma}_{0}$ is the only contract available, the consumer will happily search in lieu of accepting $\hat{\sigma}_{0}$ or exiting the market.

Expected Firm Profits. Noting the consumers' search behavior we can compute the expected profits associated with each of the offered contracts. These are given by Lemma 5 above. It is straightforward to confirm that given the definition of prices and (A.7), $\Pi_{j}\left(\sigma_{0}\right)=\Pi_{j}\left(\hat{\sigma}_{0}\right)=\Pi_{j}\left(\sigma_{1}\right)=\Pi_{j}\left(\hat{\sigma}_{1}\right)$. Therefore, each contract in the support of $\psi_{j}^{*}$ yields the same (strictly-positive) expected profit conditional on the consumers' search strategy.

No Firm has a Profitable Deviation. It remains to verify that no alternative contract can yield a greater expected profit to a typical firm given agents' search behavior and the strategy adopted by the other firms. Verification of this fact relies on the inequalities and equalities summarized by the following conditions:

$$
\begin{array}{r}
u\left(\sigma_{0} \mid \underline{v}\right)>u\left(\sigma_{1} \mid \underline{v}\right)=u\left(\hat{\sigma}_{0} \mid \underline{v}\right)=u^{*}(\underline{v}) \geq \max \left\{u\left(\hat{\sigma}_{1} \mid \underline{v}\right), 0\right\}, \text { and } \\
u\left(\sigma_{1} \mid \bar{v}\right)>u\left(\hat{\sigma}_{1} \mid \bar{v}\right)=u\left(\sigma_{0} \mid \bar{v}\right)=u^{*}(\bar{v})>u\left(\hat{\sigma}_{0} \mid \bar{v}\right) .
\end{array}
$$

(A.15) and (A.16) were derived above in relation to each type of consumers' search strategy.

Suppose firm $j$ posts the contract $\tilde{\sigma}=\langle 0, \tilde{p}\rangle$. Clearly, if $\tilde{p} \leq p_{0}$, the firm cannot improve its expected profits. The lower price fails to attract more consumers than $p_{0}$ and leads to strictly lower profits. If $\hat{p}_{0}<\tilde{p}$, then $u^{*}(\bar{v}) \geq u^{*}(\underline{v})=z-\hat{p}_{0}>z-\tilde{p}$. Thus, the more expensive contract would not be accepted by any consumers. Finally if $p_{0}<\tilde{p}<\hat{p}_{0}$, then $z-\tilde{p}<u^{*}(\bar{v})$. Hence, the contract would only be acceptable to type- $\bar{v}$ consumers. However, $\hat{\sigma}_{0}$ was also acceptable to such consumers and it had a higher price. Hence, firm $j$ could do better by offering $\hat{\sigma}_{0}$ instead.

Suppose a firm posts $\tilde{\sigma}=\langle 1, \tilde{p}\rangle$. If $\tilde{p}<p_{1}$, the firm can strictly improve its expected profit by charging a slightly higher price since the contract would be accepted by all consumers. Likewise if $\tilde{p}>\hat{p}_{1}$, the contract is not acceptable to any consumers - even type- $\bar{v}$ consumers will search in this case. If $p_{1}<\tilde{p}<\hat{p}_{1}$, the contract is acceptable only to type- $\underline{v}$ consumers. But $\hat{\sigma}_{1}$ would yield a greater profit as it would be accepted by the same number of consumers but charge them a higher price. Thus, no firm can deviate profitably from $\psi^{*}$. 


\section{B Increasing Search Costs}

In this section we elaborate on our proposed model extension with increasing search costs. Suppose that the cost to learn the contract offer of the $t$-th additional firm is $s_{t}$ where $0<s_{1} \leq s_{2} \leq \cdots$. The sequence of search costs is common knowledge and $\lim _{t \rightarrow \infty} s_{t}>\bar{v}$. If the consumer searches for $t$ periods in total, his payoff will be $u\left(\sigma_{i+t} \mid v_{i}\right)-\sum_{k=1}^{t} s_{k}$ if he accepts $\sigma_{i+t}$ and $-\sum_{k=1}^{t} s_{k}$ otherwise.

Let $\sigma_{0}=\langle 0, z\rangle$ and $\sigma_{1}=\langle 1, \underline{v}\rangle$. Suppose firms follow a strategy $\left(\psi^{*}\right)$ where $\sigma_{1}$ is offered with probability $\lambda_{1}^{*} \in(0,1)$ and $\sigma_{0}$ is offered with probability $1-\lambda_{1}^{*}$. A type- $\bar{v}$ consumer will search for $\sigma_{1}$, but only for a finite number of periods. If he searches for $t^{*}$ periods at most, $t^{*}$ must satisfy

$$
t^{*}=\max \left\{t: \lambda_{1}^{*} u\left(\sigma_{1} \mid \bar{v}\right)+\left(1-\lambda_{1}^{*}\right) u\left(\sigma_{0} \mid \bar{v}\right)-s_{t} \geq u\left(\sigma_{0} \mid \bar{v}\right)\right\} .
$$

The expected payoff from taking the final sample must exceed the payoff of accepting the available contract. We can characterize this consumer's search strategy by a decreasing sequence of cutoff values. As search costs rise over time, the consumer becomes less choosy. In period $t$ consumer $i$ will accept $\sigma_{i+t}$ if $u\left(\sigma_{i+t} \mid \bar{v}\right) \geq u_{t}^{*}(\bar{v})$ where

$$
u_{t}^{*}(\bar{v})=\left(1-\left(1-\lambda_{1}^{*}\right)^{t^{*}-t}\right) u\left(\sigma_{1} \mid \bar{v}\right)+\left(1-\lambda_{1}^{*}\right)^{t} u\left(\sigma_{0} \mid \bar{v}\right)-\sum_{k=0}^{t^{*}-t-1} s_{t^{*}-k}\left(1-\lambda_{1}^{*}\right)^{t^{*}-t-1-k} .
$$

These cutoff values can be computed via backward induction starting at $t^{*}$.

The preceding discussion has taken $\lambda_{1}^{*}$ as given. However, it is determined endogenously through the equilibration of firms' profits. Given consumers' search strategy, the expected profit of a firm offering $\sigma_{0}$ is $\Pi_{j}\left(\sigma_{0}\right)=\left(1-\gamma+\gamma\left(1-\lambda_{1}^{*}\right)^{t^{*}}\right) z$. The term $\gamma\left(1-\lambda_{1}^{*}\right)^{t^{*}}$ accounts for the small, but positive, probability that a type- $\bar{v}$ consumer accepts $\sigma_{0}$ after a $t^{*}$-period unsuccessful search for $\sigma_{1}$. Similarly,

$$
\Pi_{j}\left(\sigma_{1}\right)=\left(1+\gamma\left(\frac{1}{\lambda_{1}^{*}}-1\right)\left(1-\left(1-\lambda_{1}^{*}\right)^{t^{*}}\right)\right)(\underline{v}-c)-\phi .
$$

In equilibrium, $t^{*} \in \mathbb{N}$ and $\lambda_{1}^{*} \in(0,1)$ are such that (i) $t^{*}$ satisfies condition (B.1), and (ii) $\Pi_{j}\left(\sigma_{0}\right)=\Pi_{j}\left(\sigma_{1}\right)$. Additionally, firms must not have an incentive to deviate from $\psi^{*}$. Hence a collection of additional constraints, analogous to those in Theorem 3 but now accounting for the consumers' new search strategy, must be satisfied. ${ }^{3}$ The following lemma identifies sufficient conditions ensuring that $t^{*}$ and $\lambda_{1}^{*}$ exist in the defined environment.

Lemma B.1. Suppose search costs are non-decreasing. Define the functions

$$
\begin{aligned}
\rho(\lambda) & = \begin{cases}\max \left\{t: \lambda(\bar{v}-\underline{v})-s_{t} \geq 0\right\} & \text { if }\left\{t: \lambda(\bar{v}-\underline{v})-s_{t} \geq 0\right\} \neq \emptyset \\
0 & \text { otherwise }\end{cases} \\
\tau(\lambda) & =\frac{\log \left(1-\frac{1}{\gamma} \frac{\lambda(c+\phi-v+z)}{(\lambda-1)(c-v)+\lambda z}\right)}{\log (1-\lambda)}
\end{aligned}
$$

\footnotetext{
${ }^{3} \mathrm{As}$ in the case of the other extensions we analyze, we omit analyzing these conditions for brevity.
} 
Let $\bar{\lambda}=\frac{\gamma(\underline{v}-c)}{\phi-(1-\gamma)(\underline{v}-c-z)}$ and suppose there exists $\hat{\lambda} \in(0, \bar{\lambda})$ such that $\rho(\hat{\lambda})>\tau(\hat{\lambda})$. Then there exists $t^{*} \in \mathbb{N}$ and $\lambda_{1}^{*} \in(0,1)$ such that

1. $t^{*}=\max \left\{t: \lambda_{1}^{*} u\left(\sigma_{1} \mid \bar{v}\right)+\left(1-\lambda_{1}^{*}\right) u\left(\sigma_{0} \mid \bar{v}\right)-s_{t} \geq u\left(\sigma_{0} \mid \bar{v}\right)\right\} ;$ and,

2. $\left(1+\gamma\left(\frac{1}{\lambda_{1}^{*}}-1\right)\left(1-\left(1-\lambda_{1}^{*}\right)^{t^{*}}\right)\right)(\underline{v}-c)-\phi=\left(1-\gamma+\gamma\left(1-\lambda_{1}^{*}\right)^{t^{*}}\right) z$.

Proof. When $\sigma_{0}=\langle 0, z\rangle$ and $\sigma_{1}=\langle 1, \underline{v}\rangle, u\left(\sigma_{1} \mid \bar{v}\right)=\bar{v}-\underline{v}$ and $u\left(\sigma_{0} \mid \bar{v}\right)=0$. Now, $\rho(\lambda)$ is a non-decreasing step function taking on integer values. It is the blue curve is Figure B.1.

The function $\tau(\lambda)$ is defined as the value of $\tau$ that solves

$$
\left(1+\gamma\left(\frac{1}{\lambda}-1\right)\left(1-(1-\lambda)^{\tau}\right)\right)(\underline{v}-c)-\phi=\left(1-\gamma+\gamma(1-\lambda)^{\tau}\right) z
$$

When a solution exists, it is given by (B.3). $\tau(\lambda)$ is strictly increasing and continuous. Also, $\lim _{\lambda \rightarrow 0^{+}} \tau(\lambda)=\frac{z-\underline{v}+\phi+c}{\gamma(\underline{v}-c)}$ and $\tau(\lambda)$ asymptotes to infinity as $\lambda \rightarrow \bar{\lambda}^{-}=\frac{\gamma(\underline{v}-c)}{\phi-(1-\gamma)(\underline{v}-c-z)}$. $\tau(\lambda)$ is the red curve in Figure B.1.

If $\rho(\hat{\lambda})>\tau(\hat{\lambda})$ there will exist some $\lambda_{1}^{*} \in(\hat{\lambda}, \bar{\lambda})$ where $t^{*}=\tau\left(\lambda_{1}^{*}\right)$ and $\left(\lambda_{1}^{*}, t^{*}\right)$ satisfies the lemma's conditions. 


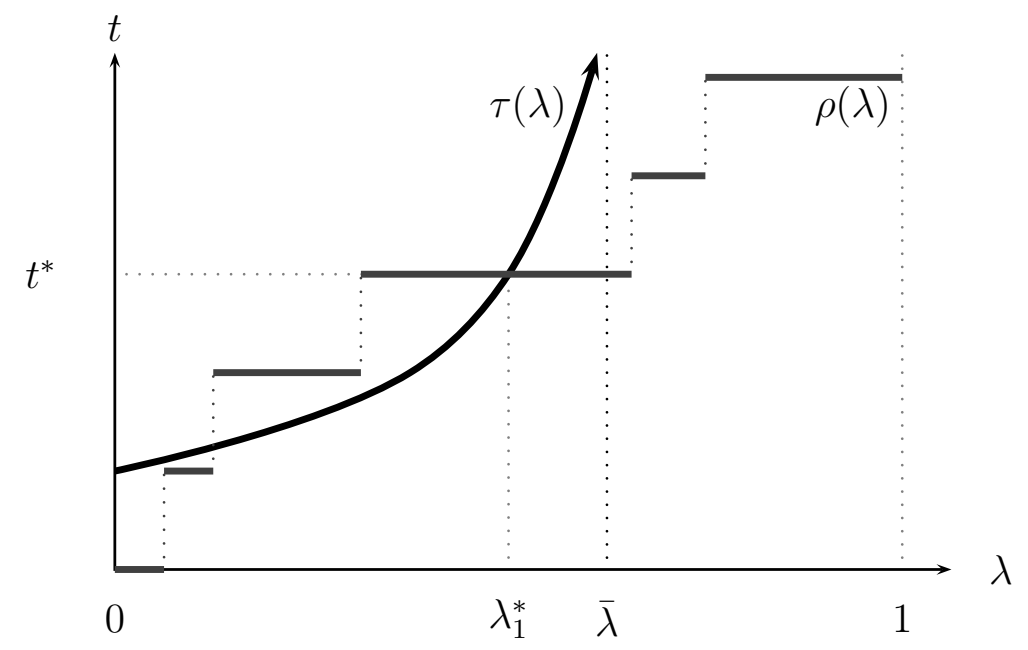

Figure B.1: An illustration of the situation in Lemma B.1.

\section{References}

DeGroot, M. H. (1970). Optimal Statistical Decisions. McGraw-Hill Book Company, New York. 